\title{
Optimization and Simulation of Labor Resource Management Information Platform Based on Internet of Things
}

\author{
Gaoyang Liang, ${ }^{1}$ Peng Cao, ${ }^{2}$ and Yang Liu $\mathbb{D}^{2}$ \\ ${ }^{1}$ School of Labor Economics, Capital University of Economics and Business, Beijing 100070, China \\ ${ }^{2}$ Hui Hua College of Hebei Normal University, Shijiazhuang, Hebei 050051, China
}

Correspondence should be addressed to Yang Liu; liuyang0809@mail.hebtu.edu.cn

Received 18 May 2021; Revised 8 July 2021; Accepted 12 July 2021; Published 26 July 2021

Academic Editor: Zhihan Lv

Copyright ( 2021 Gaoyang Liang et al. This is an open access article distributed under the Creative Commons Attribution License, which permits unrestricted use, distribution, and reproduction in any medium, provided the original work is properly cited.

\begin{abstract}
This paper conducts an in-depth analysis and research on the optimization of the labor resource management information platform through the Internet of Things (IoT) technology; through the collection, classification, and data search functions of this application system, it meets the supply and demand of professional talents within a certain enterprise. At the same time, it also realizes the curriculum training application on improving the skills and literacy of the employees of a certain enterprise, and it can learn the enterprise curriculum training from the comments of the employees on the enterprise curriculum. The effect of the enterprise course training can be learned from the comments of the employees on the enterprise course, providing an important reference basis for the future revision of the enterprise course training content. The performance of the participants in the training also has objective data for reference, so that the situation will not be disconnected from reality, and the interaction between enterprise management and employees can achieve a balanced effect. The goal of this workforce resource management system is to create a systematic workforce resource management platform for professional talents and help enterprises achieve the goal of speeding up and increasing efficiency. The system interface provided by the third party is used for horizontal data expansion to realize the sharing of basic information or video data as well as system expansion to realize real-time monitoring and management of project works. The cloud platform realizes efficient management and scientific application of construction site projects by construction management departments, which better solves the current problem of lack of supervision at construction sites.
\end{abstract}

\section{Introduction}

In recent years, with the rapid development of the Internet of Things (IoT) industry, IoT devices have shown explosive growth and have penetrated various industry sectors of society, greatly changing people's lifestyles [1]. The development of the new economy tends to have higher requirements for the overall quality and skills of the workforce. In the process of new economic development, new technologies are widely used in various new industries as the underlying factors of production, and the process of technological progress is inevitably accompanied by a gradual increase in the demand for high-skilled labor, which requires the labor supply side to make corresponding changes as the structure of labor market demand changes. With the continuous optimization and upgrading of industrial structure, the contradiction between labor supply and demand has become increasingly prominent, which is mainly manifested in the shortage of skilled talents, the imbalance between labor supply and demand regions, the prominent structural contradiction in employment, etc. The employment structure of the labor force lags the development of the industrial structure to a certain extent, and there is an incongruity between the two [2]. Under such a realistic background, it is especially important to study the influence of the new economy and new industry on labor supply and demand. The rapid development of a new economy and new industry plays an important role in adjusting the economic structure and cultivating new dynamics of economic growth and brings opportunities and challenges for employment and entrepreneurship [3]. It is 
of great theoretical value and practical significance to study the influence of new economic models on labor supply and demand.

The article uses the theory of structural unemployment, the theory of the organic composition of capital, the theory of labor resource allocation, and the theory of labor-capital in analyzing the changes affecting the supply and demand in the labor market and draws on the experience of alleviating the contradiction between labor supply and demand, based on which relevant countermeasures are proposed [4]. Through the analysis of labor market supply and demand in the new economy, this selection further enriches the development of labor market theory and has important theoretical significance for the formulation of labor market policies to achieve higher quality and fuller employment and high-quality economic development. In the process of development, the new economic model has formed a different employment model from the traditional model, and many new employment forms have emerged, and the jobs created are more flexible [5]. New changes have emerged in the relationship between supply and demand in the labor market, and the structure of supply and demand in the labor market has been affected as a result. At present, the structural contradiction between supply and demand in the labor market is prominent, which is manifested by the coexistence of "shortage" and "surplus" of labor, with structural characteristics of economic development. To correctly understand the impact of new technologies, new modes, and new business models on labor supply and demand, and to properly handle the relationship between supply and demand in the labor market, is conducive to solving the employment problem and achieving sustainable economic development [6]. The paper analyzes the impact of new economic models on labor supply and demand and proposes reasonable adjustment and optimization of industrial structure, removal of institutional barriers to labor flow, deepening reform of labor resource supply side, and establishment of the unified and standardized labor market given the problems in labor market supply and demand, which is important for transforming economic development mode and realizing comprehensive and sustainable economic development. This is important for transforming the economic development mode and achieving comprehensive and sustainable economic development.

The quality of the construction site is supervised through the mobile terminal, which can record the construction progress in real-time and report the problems encountered to the superior in time, so that the manager can assign the work to the staff and supervise the rectification, and can also distinguish the definition of responsibility to ensure the smooth implementation of quality management. In addition, the "Internet+" quality platform management system has no requirements on the distance of transmission, so that it is convenient for managers to monitor the construction site; even if the managers are in the field, they can also intuitively see the situation of the construction site, so that the supervisory departments at all levels can grasp the status of the construction site in real-time at anytime and anywhere, and remotely monitor the construction site situation and the construction progress situation, to improve efficiency and guarantee quality.

\section{Literature Review}

Zhang et al. argue that new technological changes have a huge impact on the economy and society [7]. Each new technological change and application will cause a qualitative change in the social production field, prompting the development of production methods from simple collaboration to a new division of labor, which will continuously change the form of human labor and eventually have a great impact on the existing labor market supply and demand structure [8]. The labor demand structure is influenced by the change of industrial structure to a large extent, and the change of labor demand structure requires the labor supply structure to make an adaptive adjustment [9]. However, the slow change of labor supply structure leads to the mismatch of labor market supply and demand and the imbalance between industrial structure and employment structure, which increases the employment pressure. Cao et al. pointed out that the imbalance of regional allocation of the labor force is prominent [10]. Specifically, due to the large differences between rural and urban areas in terms of infrastructure and social and public services, the development of urban and rural areas is seriously unbalanced, and the phenomenon of one-way flow of labor resources from rural areas to urban areas is obvious, which accelerates the unbalanced allocation of labor resources between urban and rural areas [11]. The above scholars have comprehensively analyzed the reasons for the contradiction between supply and demand in the labor market. First, talent development is the premise of talent development governance, and talent development governance is the advanced stage of talent development research. In-depth sorting around talent development is the only way to grasp the current challenges facing talent development and the problems more accurately that need to be solved and then try to judge the future direction of talent development governance [12]. Secondly, talent development governance is an important part of national governance, and the modernization of talent development governance is an important part of the modernization of national governance capacity and governance system. As the core concept of this study, a literature inventory of talent development governance can help grasp the current research focus, grasp the frontier theories and innovative ideas, "stand on the shoulders of giants," and cognize the functional status, core elements, and directional path of the idealized talent development governance system at a high level, to ensure a scientific and standardized. It ensures the realization of a modern talent development governance system that is scientific, standardized, open, inclusive, and efficient. When strategic labor resource management is used in corporate management, the strategy becomes the highest guiding principle for corporate orientation, which aims at pursuing sustainable competitive advantages of the company [13].

Once a strategic view of workforce management is adopted, the overall allocation of workforce resources will be weighted upward, with the company's competitive 
advantage and overall goals as the primary premise. Everyone can play a distinctive and unique role in the corporate structure [14]. A role is the expected behavior of an individual in a social organization or a specific position, but when an individual plays his or her role, he or she is influenced by the characteristics of the company and the job, personal characteristics, and the external environment and plays multiple roles at the same time [15]. In the rapidly changing and evolving era, to match the multiple evolving roles of workforce resources, this study will develop a prototype of a web-based platform that integrates various modules of workforce resources, hoping to make a small contribution to the strategic workforce resource management of an enterprise.

The Internet of Things is then included in the Japanese government's plan, mainly focusing on the basic research of precision labor and machinery research. In the actual movement of agricultural machinery, the specific data of GPS can be referred to continuously capture various images, and the terminal can achieve the expected monitoring effect. At the same time, the system can be expanded with a specialized database that meets the needs of labor output to achieve more reliable pest and disease control and harvest prediction [16]. Israel has a limited arable area due to its geography, but the Israeli government's labor force development efforts have given them a significant advantage in the world in terms of greenhouse technology. Their irrigation system uses a wide range of technologies such as sprinkler irrigation and drip irrigation, as well as sensors that identify the relevant soil moisture, effectively regulating the phase moisture environment so that crops can grow in an ideal environment. The milestone of the postindustrial economy is the emergence of the tertiary sector as the dominant economic form, also known as the knowledge economy. In the field of workforce resource management, it is even more possible to face the shock of this huge wave of the Internet head-on. The evolution of the Internet and information technology is triggering a historical change in the workforce resource management model.

\section{Optimization and Simulation Analysis of Labor Resource Management Information Platform of Internet of Things}

\subsection{IoT Labor Resource Management Information Platform} Design. In our increasingly data-centric society, IoT systems have become a vital component in generating spatiotemporal data. All aspects of various fields, from environmental monitoring to smart manufacturing, cannot be separated from the IoT. However, the key to building a complete IoT system is to solve the problem of the huge amount of data and spatiotemporal data classification of IoT spatiotemporal data [17]. For example, modern wind farms include hundreds of wind turbines, each equipped with hundreds of sensors, each with a frequency between 1 and $100 \mathrm{~Hz}$. In addition, the design and assembly of sensors are usually done by a single manufacturer, because in case of multiple manufacturers have their own models and need different configu- rations, which leads to more serious problems of sensor diversity and compatibility in the design, production, and application of practical scenarios, which leads to differences in the format of spatiotemporal data collected by IoT.

The presence and incorporation of these attributes make it necessary to address challenges such as the large volume of spatiotemporal data and real-time storage. Current IoT-based spatiotemporal data are mainly used in several important areas such as social media, healthcare, workforce, transportation, and climate science, where large amounts of spatiotemporal data are collected for spatiotemporal data processing and information search [18]. For example, the application of spatiotemporal data on transportation dynamics, the current large-scale cab pick-up, and drop-off data contains information about each trip of the cab service's customers, including the pick-up and drop-off times and locations, as well as the GPS location per second of the cab ride. This data can be used to help populations in cities be able to get cabs faster as well as overcome the effects of external factors such as traffic and weather. In addition, this data can be studied to explore traffic dynamics based on the collective movement patterns of cabs, which will help traffic authorities to be able to develop effective policies to reduce traffic congestion. In addition, this data can be used to study the behavior of cab drivers, while effective systems can be designed to detect abnormal behavior, increase the likelihood of finding new passengers, and take the best route to their destination.

At the same time, it can achieve reliable data caching support in the case of a very poor network, and when the network condition is good, the cached data can be stored to the corresponding receiving end to avoid data loss and other problems as much as possible. It fully meets the data transmission requirements of the workforce IoT management platform and is an ideal solution for pushing data from the sensing layer to the application layer in the workforce IoT management platform system. Based on these characteristics, the MQTT protocol is selected as the transmission protocol of the workforce IoT platform system, as shown in Figure 1. As shown in Figure 1, taking client 1 as an example, client 1 first sends a connection request to the MQTT server, and after a successful connection is established, client 1 publishes a message on the relevant topic to the MQTT server, and the server can achieve the expected effects of management and storage, etc. In Figure 1, the role of the sensor is to transmit the generated information.

In the case where clients 2 and 3 confirm and the connection between the Message Queuing Telemetry Transport (MQTT) server is achieved, they can subscribe to the same topic, and the MQTT server can forward the messages accordingly. The same principle can be applied to other clients for publishing. The MQTT protocol is used for data transfer, no interaction between publishers and subscribers is required, and no synchronous online communication is required; all are asynchronous; no need to know each other's IP address and port number and a series of related information so that the two sides of the communication is decoupled in space and time and. When the value is 1 , the message is persistently retained and not only sent to the current 


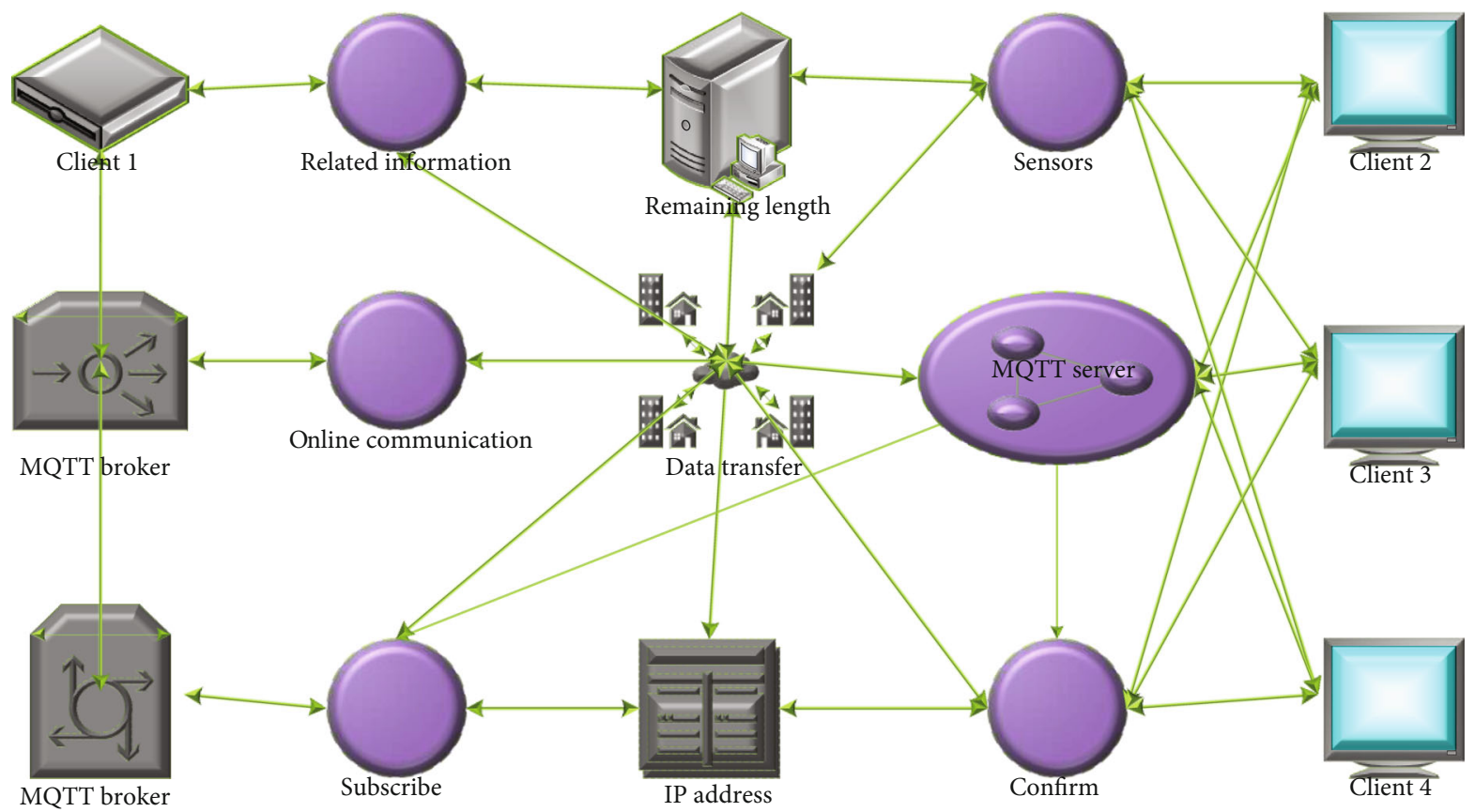

FIGURE 1: Example of MQTT protocol.

subscriber but also subscribers who subscribe to the same topic later will receive the same message when the value is 0 ; only the current message is retained and only sent to the currently subscribed subscribers.

The Remaining Length field is a two-byte representation of the number of remaining bytes, including the corresponding variable and payload content. The number of bytes in this field itself varies depending on the length of the variable header and payload. The maximum length of the Remaining Length is $256 \mathrm{~m}$. The object-oriented design approach is a revolutionary new model of industry announced to the industry. It is a holistic solution that will enable the industry to develop into a truly economically efficient industrial system that can effectively solve the crisis. The concept of object-oriented programming is to bring the principle of modularity to the fullest, with the goal of promoting professional division of labor in the industry as its mission.

The general user needs are simple interface, familiar operation methods, and the principle of human habits, and the graphical presentation of the theme makes the user feel more intimate. It does not need complicated operation function items and can meet the operation needs of all levels of employees with the most simple and practical algorithm and interface $[19,20]$. Before the system management of an enterprise, there were many paper files of labor resources. On the one hand, it is necessary to ensure that the information of labor resources will not be leaked, and on the other hand, it is necessary to ensure that the system can recover the data and operation quickly in case of damage. At the same time, technically, we must strengthen the control by adopting technically antileakage control such as document reading and writing, printing, screen copying, copying, dragging, dropping, and data transmission encryption, and in management, we must adopt multilayer security system mea- sures such as user permission and access path restriction. In addition, we should also routinely carry out work monitoring and use IP restrictions on high-frequency access to keep the system in a highly operable and secure environment, as shown in Figure 2. When logging into the system for regular users and system administrators, new users must currently register and register successful visitors to use the workforce resource system. Every time you log into the system, you need to verify your identity, i.e., you need to enter your user's name and password. The user's name can be English or numeric, and only when the user's name and password are entered correctly can the user enter the main interface of the system, and the corresponding operation page will appear.

There will be a reminder function when you make an error, and if you make five consecutive errors, your account will be temporarily locked. The core of personnel data management is security and confidentiality, and the management content covers the management of employee file information, the management of changes in the transfer and departure, the management of salary and benefits enjoyed by employees, the management of reward and punishment records, the management of training records, the management of insurance cost standards, the management of the definition of personnel data, the management of various types of employee accounts in the system, and the management of the records of complaints received and other functions.

Based on the analysis of the actual demand of labor resources, to further clarify the composition of the database and make specifications for the storage of test data later, the structure of each table of the database is listed here, as Table 1.

In the analysis and design of a workforce resource management platform, the programming language plays a pivotal 

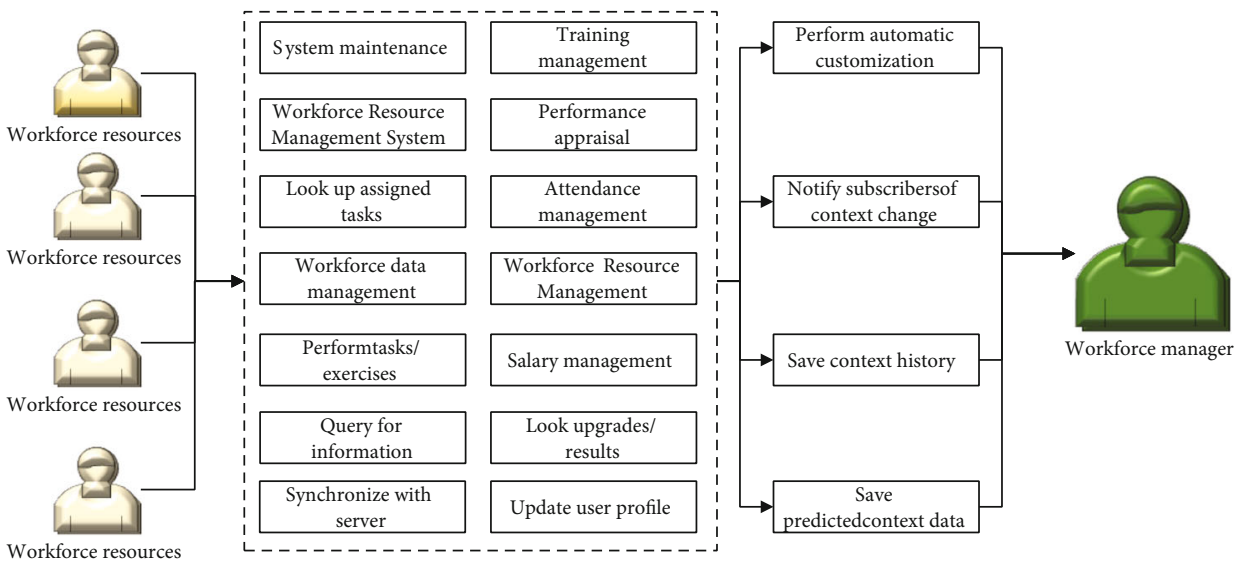

FIgURE 2: Overall system use case diagram.

TABLE 1: User information table.

\begin{tabular}{lcccc}
\hline Column name & Type & Length & Empty & Field description \\
\hline BS & Variable-length character string & 40 & No & Username \\
YHM & Variable-length character string & 40 & No & Password \\
MM & Variable-length character string & 40 & No & Employee identification number \\
YGBSH & Variable-length character string & 40 & No & Role identification \\
JSBS & Tinyint & 40 & Yes & Credit mark \\
\hline
\end{tabular}

role, and the communication between humans and machines depends entirely on the programming language to perform this complex action. The positioning of the programming language in a system is like the positioning of blood in the human body, without which the system cannot function at all. The smooth flow of blood in the human body will have a great impact on a person's health; similarly, whether a programming language can fully perform its function in a system will also have a decisive effect on the information system.

Therefore, in terms of programming language, this study did not just write code in the original PHP but used the CakePHP development framework to build the prototype of this workforce resource management system platform; taking advantage of its MVC modular architecture, the system was forced to form itself with a complete modular application architecture at the beginning of the design development. A system object is an inevitable member of it, and the objectoriented concept and its benefits are brought into full play in the MVC architecture. With a strong development team and community behind it, you can enjoy the world's most advanced web development technology with a single network line, and the infrastructure will be upgraded and revised over time. If you encounter a problem that cannot be solved, you can still seek the assistance of the development team or community on the Internet, so that the system created by the institute will not become a canoe, which will be of great help to the maintenance of the system in the future. Based on the concept of object-oriented system design, we do not need to bother to design a set of the object-oriented system from the beginning to the end, just follow the rules of this framework to build a solid object-oriented system.
Once the four main controllers in this study follow this naming convention, CakePHP will automatically find the corresponding singularly named model and its datasheet. There are many benefits to following CakePHP's naming rules, and system development should make use of such a mechanism as much as possible, so that the entire management system has a consistent style after development, increasing the readability of the code and simplifying future maintenance work. CakePHP also allows this, but it will increase the complexity of the code and should be avoided. On the user side, according to the current general computer hardware and software, it is sufficient to browse the labor resource website built by this study smoothly and use all the functions of the system. Therefore, there is no special need to install and set up the system on the user side, which is also the advantage of the WEB application.

\subsection{Platform Optimization for Simulation Experiment} Design. As the IoT real-time search system, the collected data is continuous, and usually, not much variation is found between adjacent numbers. At this time, the threshold is set to $m$; when $x_{t}-x_{t-1}>2$, here $x_{t}$ and $x_{t-1}$ belong to the same type of data. At this time, the input $x_{t}$ becomes $x_{t n}$, and $x_{t n}$ is calculated as shown in Equation (1), and $f_{t}$ is calculated at this time as shown in Equation (1).

$$
x_{t n}=\frac{\beta X_{t-2}-(1+\beta) X_{t-1}+\gamma X_{t+1}-(1+\gamma) X_{t}}{4},
$$




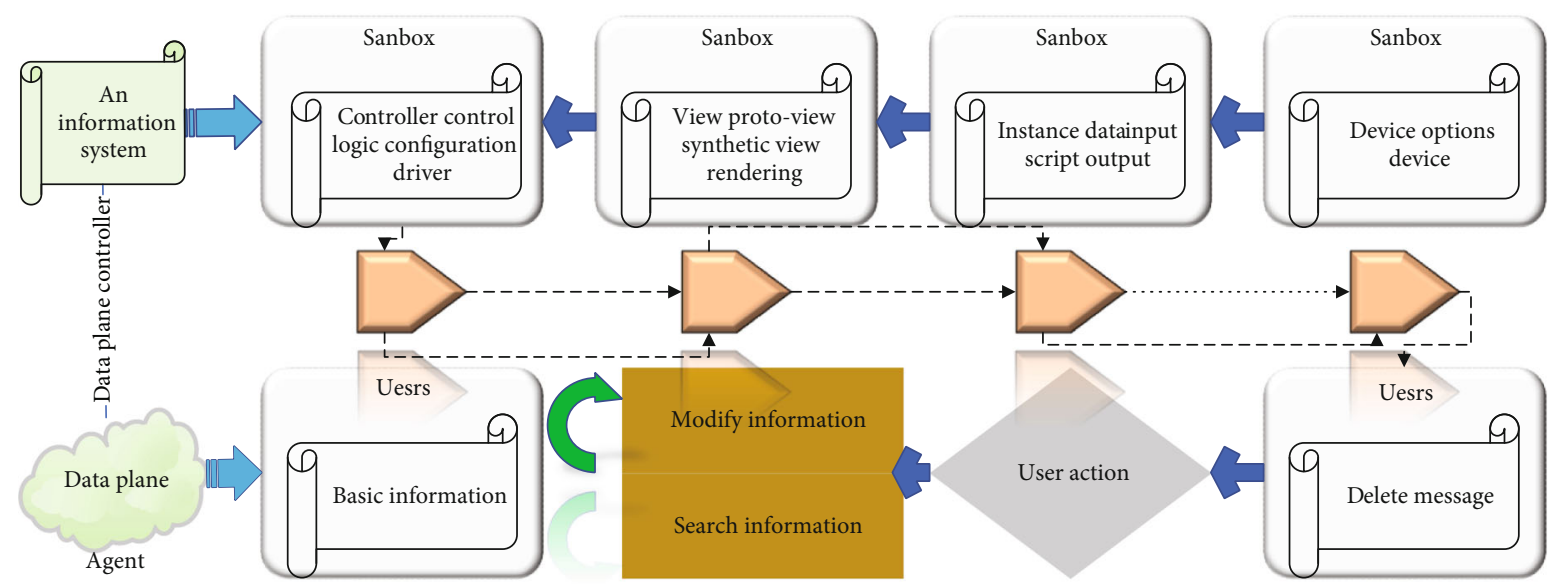

FIgURE 3: Comparison of controller and view profiles.

$$
f_{t}=\left\{\begin{array}{l}
\sigma\left(W^{f(x)} x_{t n}-W^{(f)} h_{t-1}\right), x_{t}-x_{t-1} \geq m \\
\sigma\left(W^{(f)} h_{t-1}-W^{f(x)} x_{t n}\right), x_{t}-x_{t-1}<m
\end{array}\right.
$$

$h_{t-1}$ is the hidden unit of the previous layer, $\beta$ is a constant in the interval $(0,0.5)$, and $\gamma$ denotes a constant close to 1 at infinity. $X_{t+1}$ is the information of the next spatiotemporal data collected by the IoT hardware node or sensor. The combination of the input gate and the forget gate generates a single update gate. At the same time, cell states and hidden states are also combined in the EPLSN algorithm. According to the characteristics of the data transmission of the IoT real-time query monitoring system, the data collected by a certain physical node is not very different before and after; to solve the problem and enhance the accuracy of the IoT real-time classification, the output $h_{t}$ is changed accordingly by increasing the role of $h_{t-1}$. In Equation (4), $o_{t}$ denotes the time of the center, and $c_{t}$ denotes the time of a cycle.

$$
\begin{gathered}
y=x_{t} \tanh \left(h_{t-1}\right), \\
h_{t}=o_{t} \tan \left(c_{t}\right)-y, \\
M=\frac{x_{t} \sinh \left(h_{t-1}\right)}{o_{t} \tan \left(c_{t}\right)-y} .
\end{gathered}
$$

The traditional IoT real-time search system, the IoT realtime data collection, unified transmission to a central server in a database, easy to cause data redundancy, poor system robustness, resulting in reduced search efficiency, as shown in Figure 3.

This design allows the subsystems to be presented in a structured manner, which is the focus of this study in terms of system analysis and design. View file is mainly HTML code. By properly separating the two, the program structure can be presented more clearly; instead of seeing a spaghetti-like mess of code, the program logic is separated from the display page [21-23]. This not only improves the readability of the program but also allows the target area to be locked quickly when there is a need for error removal and modification, avoiding the need to find the code to be modified, like a needle in a haystack. An information system should be designed with the possibility of follow-up maintenance in mind, and this study attempts to minimize the effort required for follow-up maintenance. Project information is collected through the collaborative platform; project managers can upload drawings, documents, and other working files to the cloud space and share them according to different permissions, and managers of all parties can access and edit documents and drawings by logging in through their accounts and will synchronize the latest information, which improves the efficiency of information circulation and reduces the risk of project construction caused by unsynchronized information.

The application of IoT is very wide, like smart home, smart grid, automotive IoT, smart coordination, security, monitoring system, and so on. In short, the Internet of Things is to make all objects, as big as the national grid and as small as dust, become intelligent. What she involves includes computer, network, software, communication, and electronics technology. So, each of her applications is a huge project that requires a variety of technologies to work together. If you are very interested in IoT, you can read related books; you will find that IoT is the big trend of the future.

As the categories of construction projects are complicated, the corresponding management processes are also relatively complex [24-26]. Therefore, the important work of the collaborative office management platform is to set up customized and diversified process management templates according to the parties involved in the project and the characteristics of the approval process and then compile practical and comprehensive approval flow templates covering the approval flow of each participant such as construction, supervision, and owner. For each process feature, different process stages, process stakeholders, process editing rights, process filing paths, etc., can also be set, to unify and standardize the approval process of all parties. The task process management of the collaborative office platform is shown in Figure 4.

Based on the customized process templates in the collaborative management platform, the approval flow that used to 


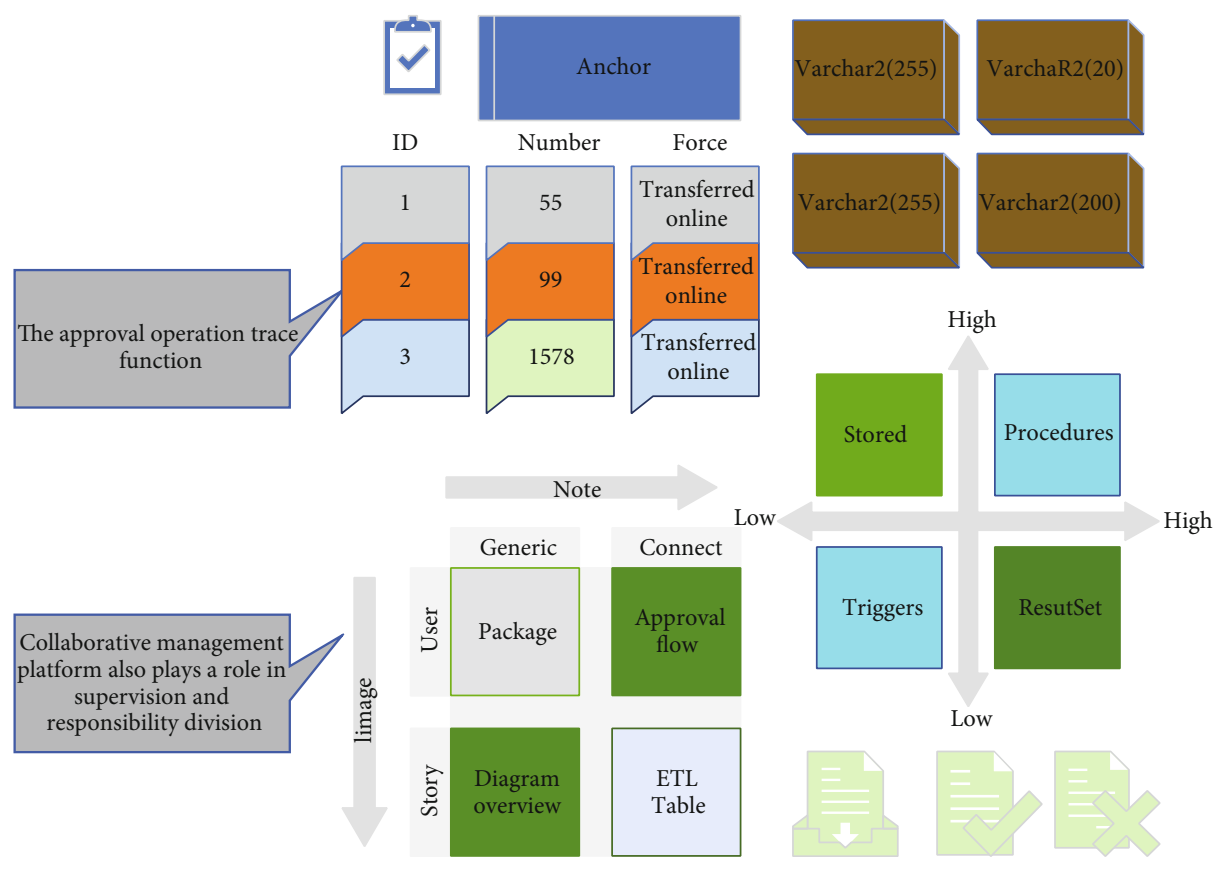

FIgURE 4: Schematic diagram of task flow management of the collaboration platform.

go offline can be successfully transferred online [27, 28]. Whether it is initiating the approval flow or processing the approval flow, it can be operated in one click on the web or cell phone, which effectively shortens the approval cycle. In addition, the approval operation trace function provided by the collaborative management platform also plays a role in supervision and responsibility division.

\section{Analysis of Results}

4.1. System Performance Test Results. The system should be thoroughly tested before delivery to find system defects and problems in the system as early as possible. This chapter first defines the testing strategy and then conducts functional and performance testing. Managers in the field are a scenario where people through the IoT have presence in the field. The system is tested by a combination of manual and automated testing. Functional test cases are designed by combining equivalence class division and boundary values, and the original test cases are reused as much as possible after repairing the functions that failed the test, and additional test cases are also needed for regression testing of the modified parts. Test the functional integrity of the system, all data types, accuracy, and function points are correct. Ensure that the system achieves the functions required by the business and that the functions accurately meet user requirements. Ensure that the system performance is up to standard, meets user requirements, and can guarantee a certain number of users running online at the same time. Using the JMeter tool to record scripts for automated testing, the stress test for this project is shown in Figure 5.

The metrics in Figure 5 show that the load-carrying capacity of the system becomes smaller as the number of con-

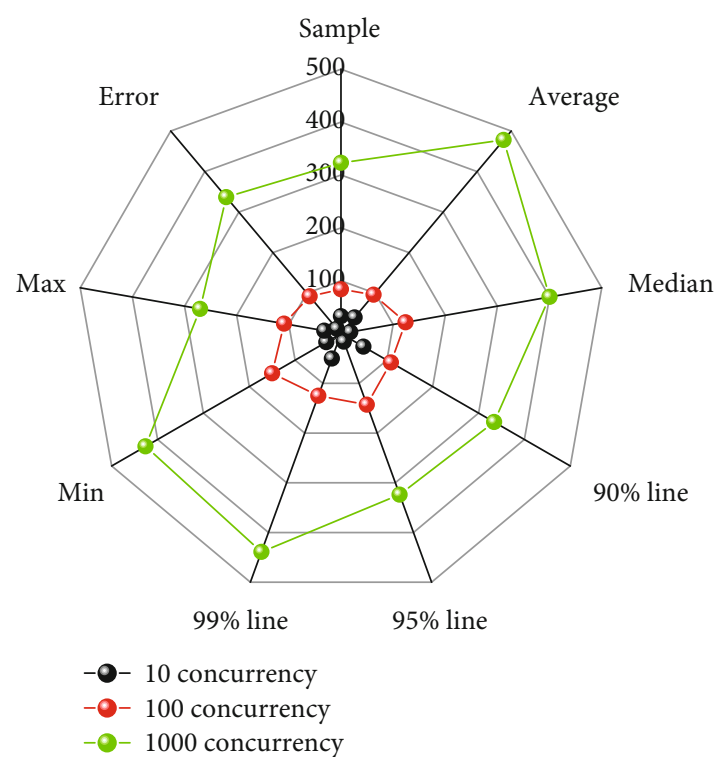

Figure 5: Stress test results.

currencies increases. When the number of concurrencies is set to 1000 , the error rate of the system increases a lot, and the response time becomes long at the same time. Therefore, it can be considered that the load capacity of the system is between 500 and 1000, which can meet the demand for concurrent user access. Through three aspects of system testing strategy, functional testing, and performance testing, a combination of manual testing and automation testing was adopted, and the original test cases were reused as much as possible after repairing the functions that failed the test, and regression testing was conducted by adding test cases for the modified parts, and the functional 


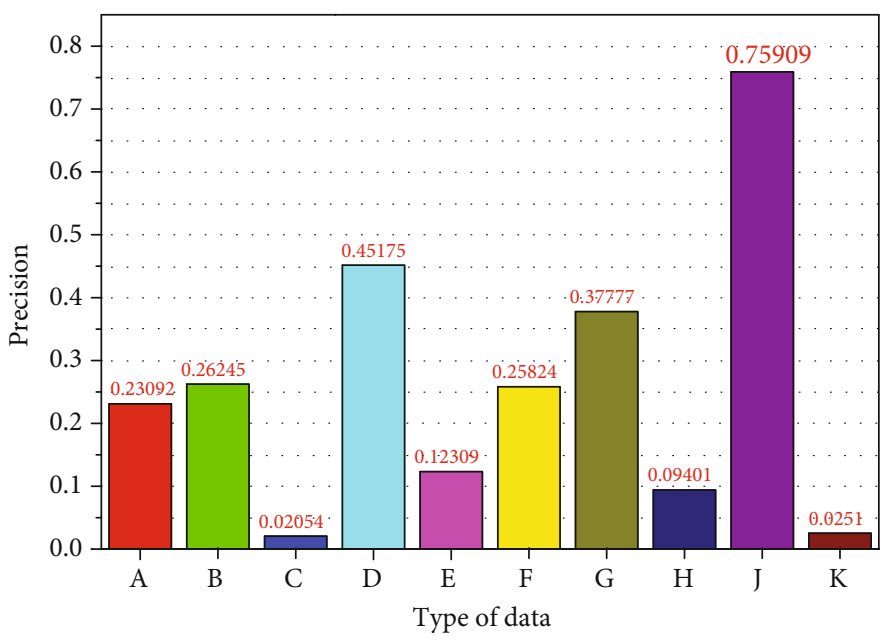

FIgURE 6: Accuracy of transmitted data.

integrity of the system was tested through automation testing by using JMeter tool to record scripts for automation testing. All data types, accuracy, and function points are correct to ensure that the system achieves the functions required by the business and that the functions accurately meet the user requirements. We ensure that the system performance meets the standards and user requirements and that a certain number of users can run online at the same time. We have achieved the objectives of the requirements of the intelligent site management system.

The payload in an MQTT message represents the message body, which is the body of the communication message and should contain additional information such as device ID and control commands, in addition to the transmission data. The data on the device side must be stored in the specified message format to carry out data communication operations that meet the requirements. After decrypting the actual message, the server will first verify the format of the data, and only the data that pass the format verification will be saved. In this section, byte type encoding is used for packet format design. The byte length of different data in the packet can be determined according to the precision range of the data, as shown in Figure 6.

Build a real-time IoT search system, the system can be through a variety of sensors including RFID, cameras, temperature, and humidity sensors and other devices for information collection; information through the encrypted secure and stable transmission server backend, the frontend personnel can query the required IoT information through this real-time IoT search system while building an Android client based on face target recognition. Through this, the client uploads the required tracking of the person photo information; the system background will automatically match the face information captured by the camera in the recent period and return the latest camera location, geographic location, and other information of the person captured to be found back to the client, to facilitate the pursuit and finding of the target. The main page of the IoT search system is based on spatiotemporal data; the system mainly

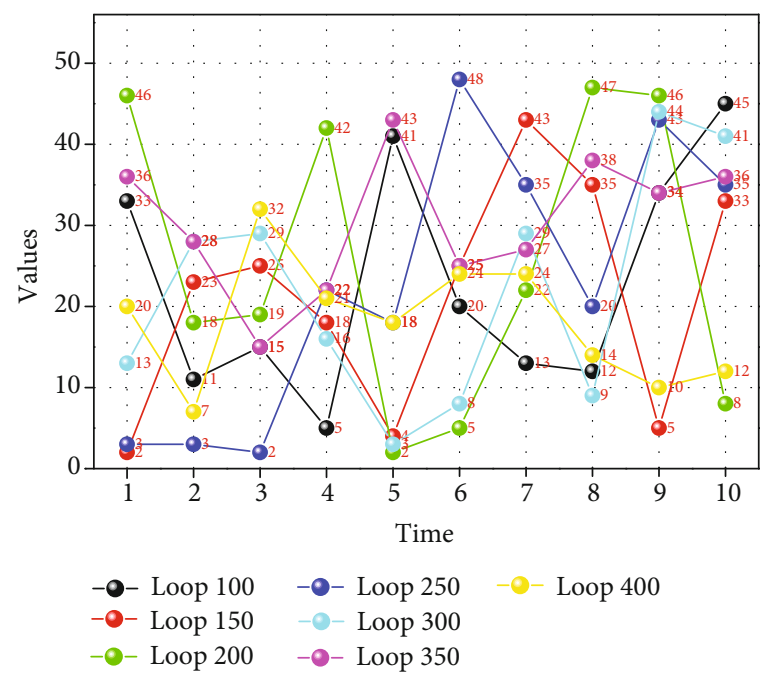

Figure 7: Ergonomics analysis.

contains a real-time video monitoring module, a face-based target tracking query module, and a search module. The first one of the message body is the encryption method value, followed by device number, device type, device status, and specific data. From Figure 6, the push time delay brought by the total number of connections is negligible with the same number of pushes, and the following conclusion can be drawn; the comprehensive performance of the server has been improved after using the hash table to optimize the subject structure of the MQTT protocol message push server, which meets the performance test requirements of the system.

4.2. Optimization and Simulation Results. At the beginning of the planning of this system, the author of this paper spent a lot of time surveying the labor resource needs of enterprises. One of the tasks is to help enterprises quickly find the talent that matches the job, determine whether the applicant meets the minimum qualification requirements needed for the job, 


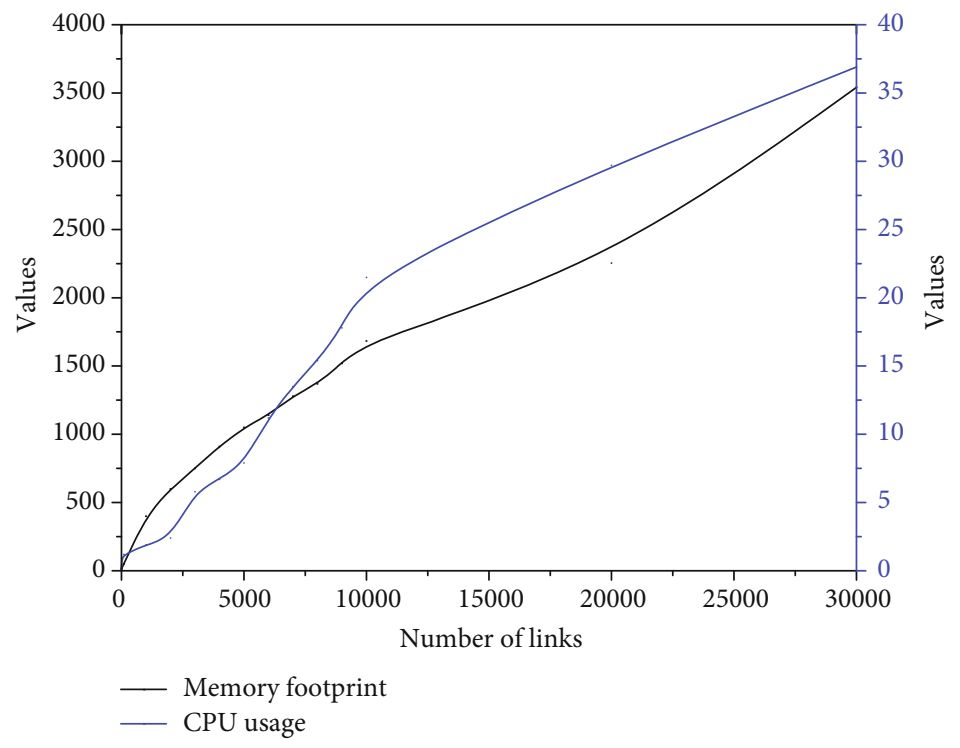

FIGURE 8: Encryption performance test results.

and help recruiters determine whether the applicant has or does not have certain attributes related to the job, and the information contained in the application form can prompt recruiters to ask potential questions related to the job. The information contained in the application form can prompt the recruiter to ask potential questions related to the job seeker in the next stage and then follow the company's scoring mechanism to determine whether this professional match the job needs or not, which is one of the important functions displayed in this workforce resource management platform. To enhance the interactivity of the corporate courses and to allow the employees to give feedback on the corporate courses, a commenting position is set up on the application, which also includes a textual position that allows the employees to express their opinions on the corporate courses anonymously at any time before, during, or after the corporate courses. The training provider is also able to use this location to express their valuable opinions on the course. The training provider can also have a comprehensive assessment of the effectiveness of its corporate courses from the messages and comments of the employees in this location, as shown in Figure 7.

For site material management, through the material management platform, the data on the receipt of various materials, the acceptance process, the amount used on the work surface, etc., are tracked and recorded, and the material tasks of each "construction unit" are checked to verify the accuracy of the planning data, whether the materials for each task are sufficient, whether there is material waste, the turnover rate of the measure materials, and whether the material loss rate exceeds the standard. Regarding whether the material wastage rate exceeds the standard, the number of materials used in each stage of the task package can be reviewed periodically through the summary of the intelligent site platform of the enterprise planning center, and appropriate adjustments can be made in time. On the other hand, through the data integration of collaborative quality management platform and material platform, the quality of material suppliers can also be evaluated, and the corresponding credit evaluation system can be formed by evaluating and analyzing the big data integrated by each material supplier in the industry, which provides data basis for the planning team to select suitable material suppliers to guarantee the quality of the project.

The system performance of the MQTT protocol using the MD5 algorithm with encryption extensions was tested during message pushing to compare how much resource consumption and pushing latency would be generated by the standard MQTT protocol, testing the pushing of 512B data, and choosing 16 bytes for the encrypted packet size; the test results are shown in Figure 8.

The test results show that when using the MD5 algorithm to encrypt and extend the MQTT protocol, the delay is less affected by the MD5 encryption compared to the network limitations because the MD5 encryption is fast and does not cause large delays to the message communication when encrypting the communication of a large amount of data transmitted by the MQTT protocol. The MVC architecture was chosen to lead the overall development of the system at the beginning of the development, in the hope that when new requirements for the application emerge in the future, new features can be added at the fastest pace to match the ever-changing real world. The optimization efficiency of our results is higher, which is about $10 \%$ higher than other studies.

Using the methods and tools of process management technology, we investigated nine information platforms in the workforce field; analyzed the causes of the three main problems found, such as low visibility, incomplete functions, and uneven quality of information content; and made suggestions for improvement. In the design process, we always consider the integration of the system and the 
optimality of the whole, and the idea of system theory runs through the design process and adheres to the PDCA cycle rule.

A complete system should have a resilient and easily expandable underlying infrastructure, so that system maintainers and developers have good specifications to follow, easy access to support teams when the system is expanded, and the ability to seek the support of global network resources with good community interaction so that the application system can obtain the latest technical support the system can keep pace with the booming development of the Internet worldwide. By clicking the location of the tracking target, the system will automatically call the Baidu map API, and the mobile system will automatically plan and search for the optimal route from the current location to the location of the tracking target so that the user can quickly achieve the target location and the pursuit and search of the target. When the query to the current location of the target for the apartment, it will automatically route planning for their location and the target location, which is convenient to quickly find the user information. Of course, since it is the route planning to the location of the camera that has taken the latest photo of the target, if there is no information of the target photo taken by the camera, there will be no query.

\section{Conclusion}

With the rapid development of artificial intelligence and IoT technology, IoT applications are becoming increasingly popular. In-depth study of MQTT protocol, design of MQTT communication module, including theme design, security mechanism, and operation configuration, improved the efficiency of data transmission. The agricultural IoT management platform is composed using Web services and frontend technology. Using the spring boot framework, the design of various aspects of the overall structure of the IoT platform, the front-end interface of the monitoring platform, and the serial communication are realized. Currently, the structural contradiction between supply and demand in the labor market is prominent and carries the structural characteristics of economic development. Finally, we propose countermeasures for solving the labor market supply and demand problems in the new economy from four aspects: rational adjustment and optimization of industrial structure, removal of institutional and mechanical obstacles to labor flow, deepening reform of labor resource supply side, and establishment of a unified and standardized labor market. Through the analysis of the labor market supply and demand in the new economy further enriches the development of labor market theory, which is of great theoretical significance to formulate labor market policies, realize the higher quality and fuller employment, and achieve high-quality economic development.

\section{Data Availability}

The data used to support the findings of this study are available from the corresponding author upon request.

\section{Conflicts of Interest}

The authors declare that they have no known competing financial interests or personal relationships that could have appeared to influence the work reported in this paper.

\section{Acknowledgments}

This work was supported by the Capital University of Economics and Business.

\section{References}

[1] H. Yao, T. Mai, J. Wang, Z. Ji, C. Jiang, and Y. Qian, "Resource trading in blockchain-based industrial internet of things," IEEE Transactions on Industrial Informatics, vol. 15, no. 6, pp. 3602-3609, 2019.

[2] H. Yang, S. Kumara, S. T. S. Bukkapatnam, and F. Tsung, "The Internet of Things for smart manufacturing: a review," IISE Transactions, vol. 51, no. 11, pp. 1190-1216, 2019.

[3] Y. Hong, L. Chen, and L. Mo, "Optimization of cluster resource indexing of Internet of Things based on improved ant colony algorithm," Cluster Computing, vol. 22, no. S3, pp. 7379-7387, 2019.

[4] C. Jian, M. Li, and X. Kuang, "Edge cloud computing service composition based on modified bird swarm optimization in the Internet of Things," Cluster Computing, vol. 22, no. S4, pp. 8079-8087, 2019.

[5] Q. Gao, S. Guo, X. Liu, G. Manogaran, N. Chilamkurti, and S. Kadry, "Simulation analysis of supply chain risk management system based on IoT information platform," Enterprise Information Systems, vol. 14, no. 9-10, pp. 1354-1378, 2020.

[6] C. Zhan and H. Lai, "Energy minimization in Internet-ofThings system based on rotary-wing UAV," IEEE Wireless Communications Letters, vol. 8, no. 5, pp. 1341-1344, 2019.

[7] K. Wang, Y. Zhou, Z. Liu, Z. Shao, X. Luo, and Y. Yang, "Online task scheduling and resource allocation for intelligent NOMA-based industrial Internet of Things," IEEE Journal on Selected Areas in Communications, vol. 38, no. 5, pp. 803-815, 2020.

[8] A. Darwish, A. E. Hassanien, M. Elhoseny, A. K. Sangaiah, and K. Muhammad, "The impact of the hybrid platform of internet of things and cloud computing on healthcare systems: opportunities, challenges, and open problems," Journal of Ambient Intelligence and Humanized Computing, vol. 10, no. 10, pp. 4151-4166, 2019.

[9] W. Hu, H. Li, W. Yao, and Y. Hu, "Energy optimization for WSN in ubiquitous power internet of things," International Journal of Computers Communications \& Control, vol. 14, no. 4, pp. 503-517, 2019.

[10] B. Cao, X. Wang, W. Zhang, H. Song, and Z. Lv, "A manyobjective optimization model of industrial Internet of Things based on private blockchain," IEEE Network, vol. 34, no. 5, pp. 78-83, 2020.

[11] Z. Ghanbari, N. Jafari Navimipour, M. Hosseinzadeh, and A. Darwesh, "Resource allocation mechanisms and approaches on the Internet of Things," Cluster Computing, vol. 22, no. 4, pp. 1253-1282, 2019.

[12] M. P. K. Reddy and M. R. Babu, "Implementing self adaptiveness in whale optimization for cluster head section in Internet of Things," Cluster Computing, vol. 22, no. S1, pp. 1361-1372, 2019. 
[13] L. Kong and B. Ma, "Intelligent manufacturing model of construction industry based on Internet of Things technology," The International Journal of Advanced Manufacturing Technology, vol. 107, no. 3-4, pp. 1025-1037, 2020.

[14] P. Kumar and L. Chouhan, "Design of secure session key using unique addressing and identification scheme for smart home Internet of Things network: NA," Transactions on Emerging Telecommunications Technologies, vol. 32, no. 5, article e3993, 2020.

[15] Z. Ma, M. Xiao, Y. Xiao, Z. Pang, H. V. Poor, and B. Vucetic, "High-reliability and low-latency wireless communication for internet of things: challenges, fundamentals, and enabling technologies," IEEE Internet of Things Journal, vol. 6, no. 5, pp. 7946-7970, 2019.

[16] J. Wan, J. Li, Q. Hua, A. Celesti, and Z. Wang, "Intelligent equipment design assisted by Cognitive Internet of Things and industrial big data," Neural Computing and Applications, vol. 32, no. 9, pp. 4463-4472, 2020.

[17] T. J. Saleem and M. A. Chishti, "Data analytics in the Internet of Things: a survey," Scalable Computing: Practice and Experience, vol. 20, no. 4, pp. 607-630, 2019.

[18] M. Ben-Daya, E. Hassini, and Z. Bahroun, "Internet of Things and supply chain management: a literature review," International Journal of Production Research, vol. 57, no. 15-16, pp. 4719-4742, 2019.

[19] S. Tian, T. Wang, L. Zhang, and X. Wu, "The Internet of Things enabled manufacturing enterprise information system design and shop floor dynamic scheduling optimisation," Enterprise Information Systems, vol. 14, no. 9-10, pp. 12381263, 2020.

[20] M. Noura, M. Atiquzzaman, and M. Gaedke, "Interoperability in internet of things: taxonomies and open challenges," Mobile Networks and Applications, vol. 24, no. 3, pp. 796-809, 2019.

[21] N. Mircică, "Cyber-physical systems for cognitive Industrial Internet of Things: sensory big data, smart mobile devices, and automated manufacturing processes," Analysis and Metaphysics, vol. 18, pp. 37-43, 2019.

[22] M. Abdel-Basset, G. Manogaran, A. Gamal, and V. Chang, "A novel intelligent medical decision support model based on soft computing and IoT," IEEE Internet of Things Journal, vol. 7, no. 5, pp. 4160-4170, 2019.

[23] J. Yang, C. Wang, B. Jiang, H. Song, and Q. Meng, "Visual perception enabled industry intelligence: state of the art, challenges and prospects," IEEE Transactions on Industrial Informatics, vol. 17, no. 3, pp. 2204-2219, 2020.

[24] J. Yang, Z. Zhang, W. Mao, and Y. Yang, "Identification and micro-motion parameter estimation of non-cooperative UAV targets," Physical Communication, vol. 46, p. 101314, 2021.

[25] W. Wei, Q. Ke, J. Nowak, M. Korytkowski, R. Scherer, and M. Woźniak, "Accurate and fast URL phishing detector: a convolutional neural network approach," Computer Networks, vol. 178, p. 107275, 2020.

[26] Y. Li and J. Yang, "Meta-learning baselines and database for few-shot classification in agriculture," Computers and Electronics in Agriculture, vol. 182, p. 106055, 2021.
[27] A. Zielonka, A. Sikora, M. Wozniak, W. Wei, Q. Ke, and Z. Bai, "Intelligent Internet of Things system for smart home optimal convection," IEEE Transactions on Industrial Informatics, vol. 17, no. 6, pp. 4308-4317, 2021.

[28] X. Lv, Y. Liu, S. Xu, and Q. Li, "Welcoming host, cozy house? The impact of service attitude on sensory experience," International Journal of Hospitality Management, vol. 95, p. 102949, 2021. 